\title{
High-order harmonic generation in polyatomic molecules induced by a bicircular laser field
}

\author{
S. Odžak, ${ }^{1}$ E. Hasović, ${ }^{1}$ and D. B. Milošević ${ }^{1,2,3}$ \\ ${ }^{1}$ Faculty of Science, University of Sarajevo, Zmaja od Bosne 35, 71000 Sarajevo, Bosnia and Herzegovina \\ ${ }^{2}$ Max-Born-Institut, Max-Born-Str. 2a, 12489 Berlin, Germany \\ ${ }^{3}$ Academy of Sciences and Arts of Bosnia and Herzegovina, \\ Bistrik 7, 71000 Sarajevo, Bosnia and Herzegovina
}

(Dated: May 27, 2022)

\begin{abstract}
High-order harmonic generation by a bicircular field, which consists of two coplanar counterrotating circularly polarized fields of frequency $r \omega$ and $s \omega$ ( $r$ and $s$ are integers), is investigated for a polyatomic molecule. This field possesses dynamical symmetry, which can be adapted to the symmetry of the molecular Hamiltonian and used to investigate the molecular symmetry. For polyatomic molecules having the $C_{r+s}$ symmetry only the harmonics $n=q(r+s) \pm r, q=1,2, \ldots$, are emitted having the ellipticity $\varepsilon_{n}= \pm 1$. We illustrate this using the example of the planar molecules $\mathrm{BH}_{3}$ and $\mathrm{BF}_{3}$, which obey the $C_{3}$ symmetry. We show that for the $\mathrm{BF}_{3}$ molecule, similarly to atoms with a $p$ ground state, there is a strong asymmetry in the emission of high harmonics with opposite helicities. This asymmetry depends on the molecular orientation.
\end{abstract}

Strong-field physics and attoscience are presently a very active area of research since they enable one to study matter on the scale of the electronic dynamics [1. The electron liberated by a strong linearly polarized laser field can return to the parent ion and trigger various strong-field processes, which include elastic rescattering (the process in which the final electron energy is much higher than in direct ionization is called high-order above-threshold ionization [2]), the inelastic process in which in an ionizing collision one or more additional electrons are liberated 3, and the laser-assisted electronion recombination process where a high-energy photon is emitted [this is the so-called high-order harmonic generation (HHG) 4]]. The dynamics of electrons driven by a linearly polarized laser field is one-dimensional and with it it is difficult to explore structure and dynamics of more complex targets such as molecules (for reviews about molecular strong-field processes see [5, 6]). Since symmetry is a fundamental concept of science and since it is the key for understanding the structure and dynamics of molecules, it is important to find field configurations that possess both particular symmetry properties and the possibility that the laser-field-driven liberated electron returns to the parent ion. Such a field will be the main subject of the present study.

The concept of symmetry is particularly relevant for polyatomic molecules. Due to the complexity of polyatomic molecules, research of molecular HHG has mainly been confined to simple diatomic and triatomic linear molecules. A list of recent papers in which polyatomic molecules were studied includes $7-18$. Ab initio HHG calculations for polyatomic molecules are too demanding and thus far have not been possible in any detail. We have recently developed a strong-field-approximation theory of HHG by polyatomic molecules [19] and illustrated it by the examples of the ozone and the carbon dioxide molecules exposed to a linearly polarized laser field. In the present work we apply this theory to more complex molecules and more complex laser fields. Our goal is to relate the respective molecular symmetry to the symmetry of the laser field and to explore the influence of these symmetries on the HHG spectra. We neglect the vibrational dynamics during the HHG process since it has recently been shown that for a broad range of molecules in strong ultrashort laser pulses it does not play an important role 18 .

A special field combination, called the bicircular field, has recently attracted a lot of attention. A bicircular field consists of two coplanar counterrotating circularly polarized fields with frequencies $r \omega$ and $s \omega$, which are integer multiples of the fundamental frequency $\omega$. In our notation this field is defined in the $x y$ plane by [20]

$$
\begin{aligned}
& E_{x}(t)=\left[E_{1} \sin (r \omega t)+E_{2} \sin (s \omega t)\right] / \sqrt{2}, \\
& E_{y}(t)=\left[-E_{1} \cos (r \omega t)+E_{2} \cos (s \omega t)\right] / \sqrt{2} .
\end{aligned}
$$

A possible phase offset of the first component is canceled by an appropriate choice of the initial time, while an offset the phase of the second component is canceled by a rotation of the field around the $z$ axis by the angle $-\phi_{2} /(r+s)$ (for $s \omega t \rightarrow s \omega t+\phi_{2}$ ).

HHG by such field has been shown experimentally to be very efficient as early as in 1995 21 and was investigated theoretically in subsequent years 22 31]. Crucial for the revival of interest in strong-field processes in bicircular fields was the experimental confirmation that the generated harmonics are circularly polarized 32. This paper has triggered a series of papers about HHG by atoms exposed to bicircular fields 3342 . Other atomic processes in strong bicircular fields were also investigated, such as strong-field ionization [43 47, laser-assisted recombination [20], nonsequential double ionization [48, the possibility of introducing spin into attoscience [49], etc. Molecular HHG using a bicircular field was studied in 50 52. 
The electric field vector of the $r \omega-s \omega$ bicircular field is invariant with respect to simultaneous translation in time by $p T /(r+s)$, where $p$ is an integer and $T=2 \pi / \omega$ is the optical period, and rotation about the $z$ axis, which is perpendicular to the polarization plane, by the angle $\operatorname{pr} 2 \pi /(r+s)$. For the $\omega-2 \omega$ field this dynamical symmetry means that $R_{z}(p 2 \pi / 3) \mathbf{E}(t+p T / 3)=\mathbf{E}(t)$, i.e. for $p=1$ the translation in time is by one third of the optical cycle and the rotation is by the angle $120^{\circ}$. If the laser-free molecular Hamiltonian is invariant under rotation by the angle $\operatorname{pr} 2 \pi /(r+s)$ then it can be shown in the same way as in Appendix A of Ref. 39. that the afore-mentioned dynamical symmetry leads to the following selection rule: only harmonics of the order

$$
n=q(r+s) \pm r, q-\text { integer }
$$

are emitted and they are circularly polarized with the helicity $\varepsilon_{n}= \pm 1$, respectively. If a molecule possesses the rotational symmetry $C_{r+s}$ then the Hamiltonian of this molecule is invariant with respect to a rotation by the angle $2 \pi /(r+s)$ about the $z$ axis, which is chosen to be the molecular quantization axis [53, 54]. For example, the molecules $\mathrm{NH}_{3}, \mathrm{CF}_{3} \mathrm{I}, \mathrm{SO}_{3}, \mathrm{BH}_{3}$, and $\mathrm{BF}_{3}$ possess the $C_{3}$ symmetry so that the Hamiltonians of these molecules are invariant with respect to rotation by the angle $p \cdot 120^{\circ}$. If these molecules are exposed to the $\omega-2 \omega$ bicircular field in the $x y$ polarization plane, the harmonics $n=3 q, q=$ $1,2,3, \ldots$, are absent from their HHG spectra, while the harmonics $n=3 q \pm 1$ are circularly polarized with the ellipticity $\varepsilon_{n}= \pm 1$.

The power of the $n$th harmonic having the wave vector $\mathbf{K}$ and the unit complex polarization vector $\hat{\mathbf{e}}_{\mathbf{K}}$ is [19, 39]

$$
P_{n}=\frac{(n \omega)^{4}}{2 \pi c^{3}}\left|\mathbf{T}_{n}\right|^{2}, \quad \mathbf{T}_{n}=\int_{0}^{T} \frac{d t}{T} e^{i n \omega t} \sum_{m} \mathbf{d}_{m}(t),
$$

where $\mathbf{T}_{n}=T_{n} \hat{\mathbf{e}}_{\mathbf{K}}=\sum_{\xi=x, y, z} T_{n \xi} \hat{\mathbf{e}}_{\xi}$ and $\mathbf{d}_{m}(t)$ is the time-dependent dipole which corresponds to the $m$ th molecular orbital of the neutral polyatomic molecule having closed shells. Within the strong-field approximation, the time-dependent dipole is given by 19

$$
\begin{aligned}
\mathbf{d}_{m}(t)= & -i\left(\frac{2 \pi}{i}\right)^{3 / 2} \int_{0}^{\infty} \frac{d \tau}{\tau^{3 / 2}} e^{-i S_{s}} \\
& \times \sum_{k=1}^{N} e^{i\left[\mathbf{k}_{s}+\mathbf{A}(t)\right] \cdot \mathbf{r}_{k}}\left\langle\varphi_{k m}|\mathbf{r}| \mathbf{k}_{s}+\mathbf{A}(t)\right\rangle \\
& \times \sum_{j=1}^{N} e^{-i \mathbf{k}_{s} \cdot \mathbf{r}_{j}}\left\langle\mathbf{k}_{s}+\mathbf{A}(t-\tau)|\mathbf{r} \cdot \mathbf{E}(t-\tau)| \varphi_{j m}\right\rangle
\end{aligned}
$$

with $S_{s} \equiv \int_{t-\tau}^{t} d t^{\prime}\left[\mathbf{k}_{s}+\mathbf{A}\left(t^{\prime}\right)\right]^{2} / 2+I_{p} \tau, \quad I_{p}$ the ionization potential, $\mathbf{k}_{s}=-\int_{t-\tau}^{t} d t^{\prime} \mathbf{A}\left(t^{\prime}\right) / \tau$, and $\mathbf{E}(t)=-d \mathbf{A}(t) / d t$. The molecular orbitals $\phi_{m}(\mathbf{r})=$ $\sum_{j=1}^{N} \varphi_{j m}(\mathbf{r})=\sum_{j=1}^{N} \sum_{a} c_{j a m} \psi_{a m}(\mathbf{r})$ are written as linear combinations of the atomic orbitals $\psi_{a m}$, which are
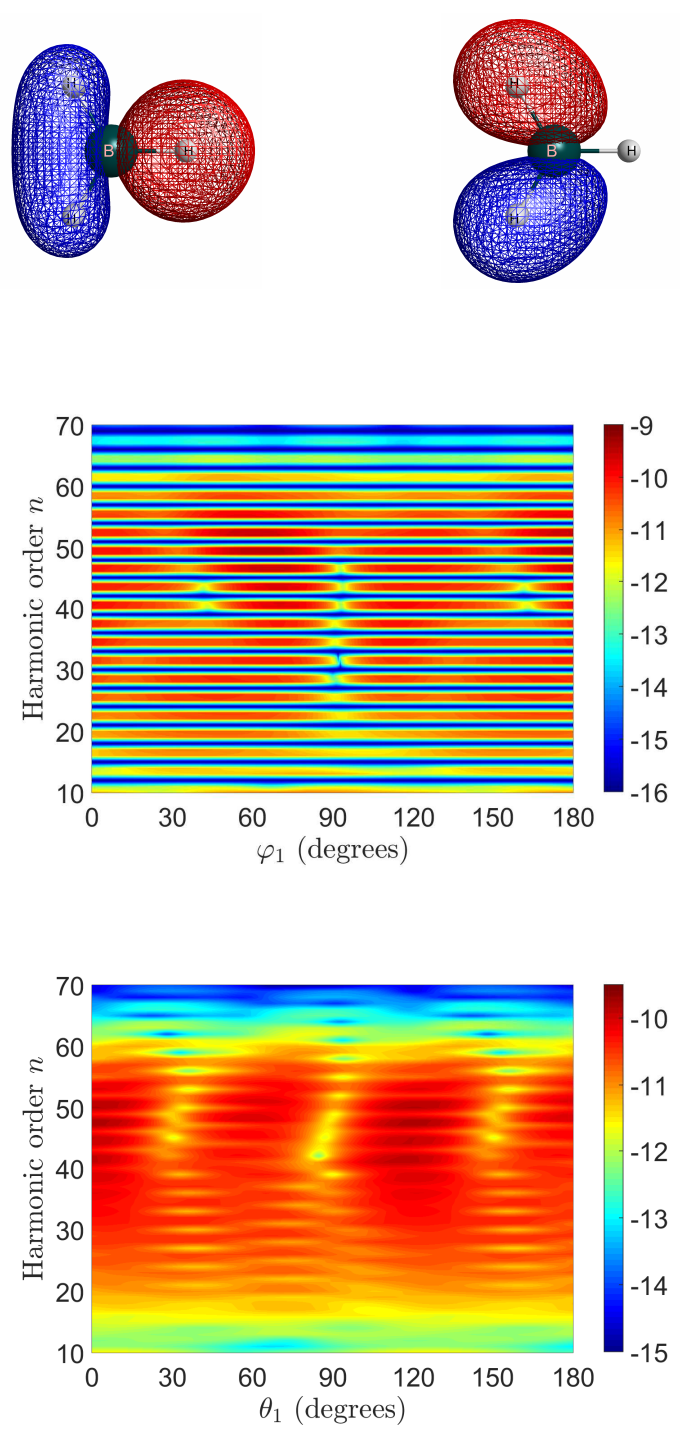

FIG. 1: (Color online) Upper panel: two degenerate HOMOs of the $\mathrm{BH}_{3}$ molecule in the $x y$ plane. Middle (lower) panel: logarithm of the harmonic power for the $\mathrm{BH}_{3}$ molecule presented in false colors as a function of the rotation angle $\varphi_{1}\left(\theta_{1}\right)$ in the $x y(x z)$ plane (see Supplementary information for the definition of this rotation). The bicircular laser field component intensities are equal $4 \times 10^{14} \mathrm{~W} / \mathrm{cm}^{2}$ and the fundamental wavelength is $800 \mathrm{~nm}$.

expressed as linear combinations of primitive Gaussian functions 53. Here $\mathbf{r}=\mathbf{r}_{e}-\mathbf{r}_{j}$, where $\mathbf{r}_{j}, j=1, \ldots, N$, are the position vectors of the atoms and $\mathbf{r}_{e}$ is the electron coordinate.

For our calculation we will use the examples of the planar $\mathrm{BH}_{3}$ and $\mathrm{BF}_{3}$ molecules (see Supplementary information), which both possess the $C_{3}$ symmetry. The highest occupied molecular orbital (HOMO) of the $\mathrm{BH}_{3}$ molecule is doubly degenerate having the ionization potential $I_{p}=13.59 \mathrm{eV}$ and the $E^{\prime}$ symmetry. The ion- 


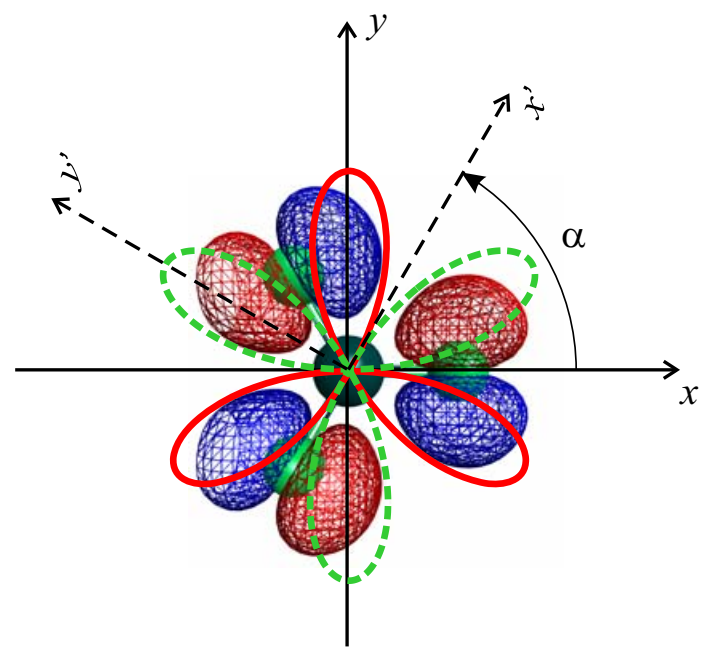

FIG. 2: (Color online) Bicircular field and its rotation in the $x y$ plane by the angle $\alpha=60^{\circ}$, presented together with the $\mathrm{HOMO}$ of the $\mathrm{BF}_{3}$ molecule.

ization energies of the other molecular orbitals are much higher and their contributions can be neglected. Therefore, in Eq. (4) we have the sum over two degenerate molecular orbitals. Even though the molecular orbitals are not symmetric with respect to rotation by the angle $120^{\circ}$ about the $z$ axis (see the upper panel of Fig. 1), the HHG spectra for the bicircular $\omega-2 \omega$ field in the molecular $x y$ plane (middle panel of Fig. 1) show that the harmonics $n=3 q$ are absent. On the other hand, if the bicircular field is not in the molecular plane the symmetry is violated and all harmonics are emitted. This is clearly visible in the bottom panel of Fig. 11 where the bicircular field rotates in the $x z$ plane.

The $\mathrm{BH}_{3}$ molecule is relatively simple and no particular structure is visible in the spectra presented in Fig. 1 . Let us now consider the $\mathrm{BF}_{3}$ molecule, which possesses the same $C_{3}$ symmetry but the $\mathrm{H}$ atoms are replaced by more complex $\mathrm{F}$ atoms. The orbitals of the $\mathrm{BF}_{3}$ molecule are closer in energy (see Table II in Supplementary Information). The orbitals denoted by a double prime (") are antisymmetric with respect to reflection [53 about the horizontal $(x y)$ plane so that the corresponding ionization matrix element in Eq. (4) is zero (for $z \rightarrow-z$ the field does not change). Therefore, HOMO-1 and HOMO3 , having, respectively, $E^{\prime \prime}$ and $A_{2}^{\prime \prime}$ symmetry, do not contribute to the harmonic emission. The HOMO of the $\mathrm{BF}_{3}$ molecule, having the $A_{2}^{\prime}$ symmetry $[53$, is presented in Fig. 2, together with the bicircular field, which is in the $x y$ plane and rotated by the angle $\alpha=\varphi_{1}=0^{\circ}$ (red solid line) and $60^{\circ}$ (green dashed line).

In Fig. 3 we present the HHG spectra for the $\mathrm{BF}_{3}$ molecule and the $\omega-2 \omega$ bicircular field in the $x y$ polarization plane. From the upper panel of Fig. 3 we see that only the harmonics $n=3 q \pm 1$ are emitted for arbitrary
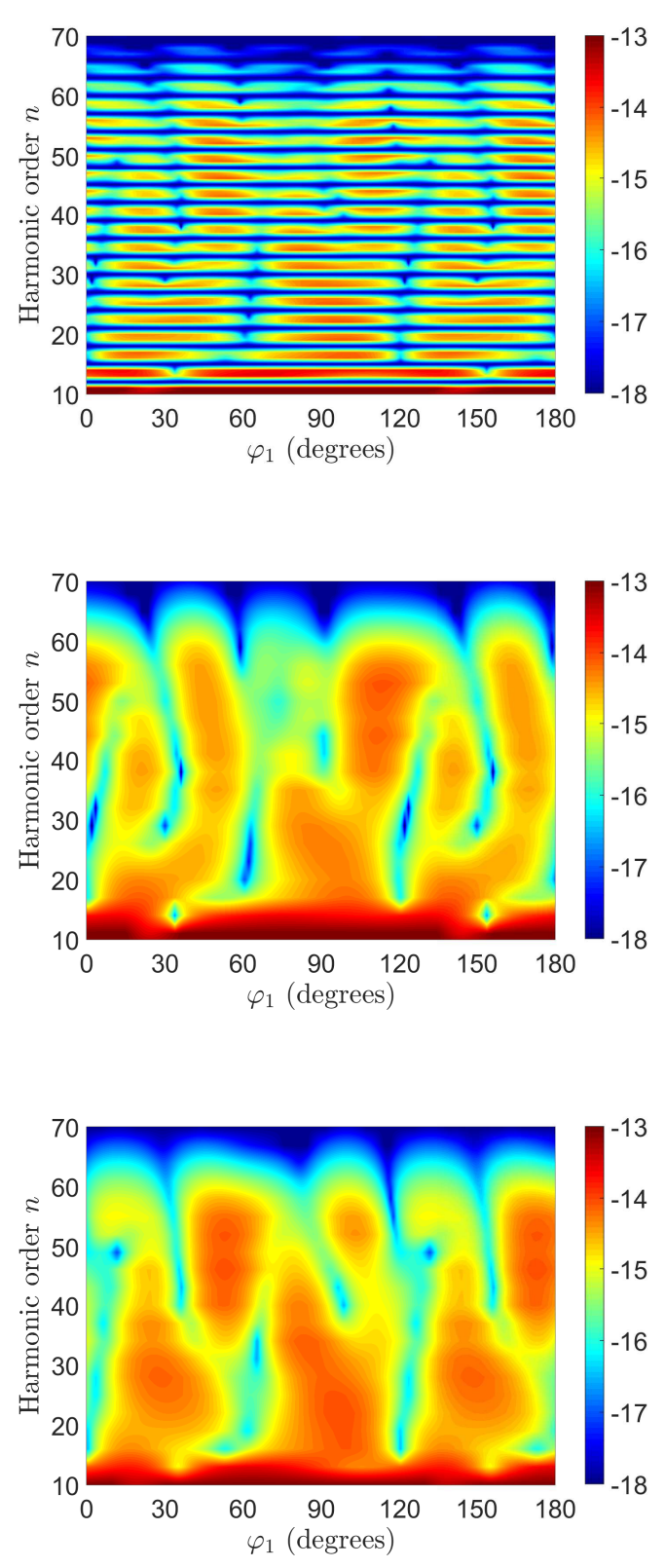

FIG. 3: (Color online) Upper panel: logarithm of the harmonic power for the $\mathrm{BF}_{3}$ molecule presented in false colors as a function of the rotation angle $\varphi_{1}$ in the $x y$ plane (horizontal axis) and harmonic order $n$ (vertical axis). Middle (bottom) panel: same as in the upper panel but with only the harmonics having ellipticity $\varepsilon_{n}=-1(+1)$. The parameters of the bicircular laser field are as in Fig. 1

rotation angle $\varphi_{1}$ in the $x y$ plane. In addition, more structures are visible in the spectrum. These structures are invariant with respect to translation by $120^{\circ}$ (the results for $0 \leq \varphi_{1} \leq 60^{\circ}$ are identical to the results for $\left.120 \leq \varphi_{1} \leq 180^{\circ}\right)$. The interference structure visible in the spectrum can be related to the shape of the HOMO. 

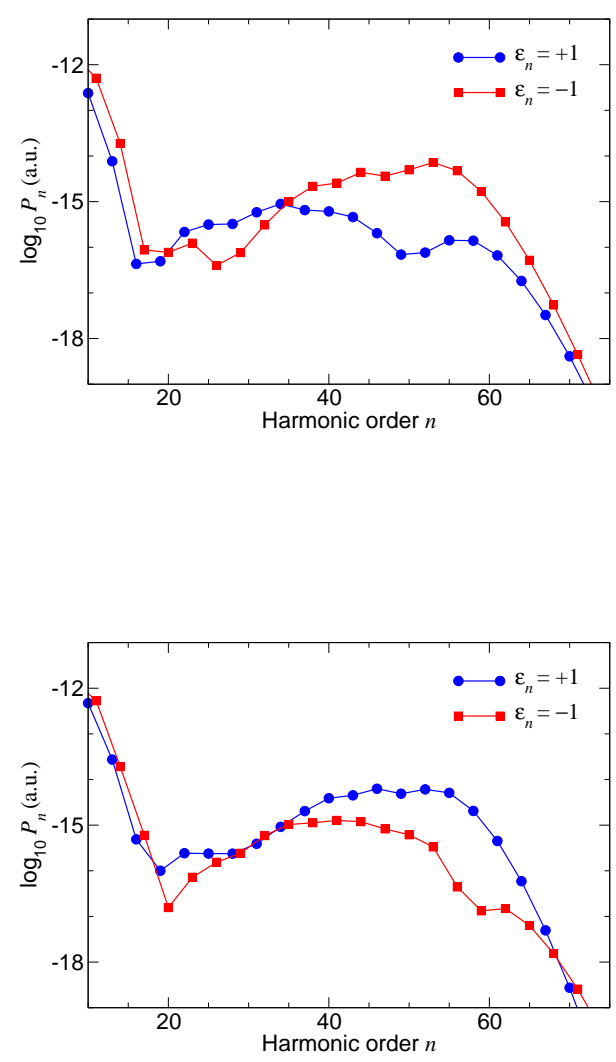

FIG. 4: (Color online) Logarithm of the harmonic power for the $\mathrm{BF}_{3}$ molecule presented as a function of the harmonic order for the same laser parameters as in Fig. 1 for the HOMO and for $\varphi_{1}=0^{\circ}$ (upper panel) and $\varphi_{1}=60^{\circ}$ (lower panel). The bicircular field is in the $x y$ plane as denoted in the upper panel of Fig. 2 The harmonic ellipticity is indicated in the legend.

From Fig. 2 we see that the polar diagram of the bicircular electric field vector for $\alpha=0^{\circ}$ partially overlaps with the "blue" part of the electron density, while for $\alpha=60^{\circ}$ it overlaps with the "red" part for which the electron wave function has the opposite sign. For different $\alpha$ this overlap is different which is the cause of the change of the interference structure.

It has been shown in Refs. 36, 39, that for atomic HHG from a $p$ ground state the yields of the $3 q+1$ and $3 q-1$ harmonics are different. Since the $\mathrm{F}$ atoms in the $\mathrm{BF}_{3}$ molecule behave as $p$-state atoms we expect that such an asymmetry will also appear for the $\mathrm{BF}_{3}$ molecule. This is clearly visible in the middle and bottom panels of Fig. 3, where the spectra of harmonics $n=3 q-1$ with $\varepsilon_{n}=-1$ (middle panel) and the harmonics $n=3 q+1$ with $\varepsilon_{n}=+1$ (bottom panel) are shown. The results are interpolated and smoothed for a better visibility of the interference structures. As a further example, in Fig. 4 we compare the $\varepsilon_{n}=+1$ and $\varepsilon_{n}=-1$ spectra for two fixed values of the angle $\varphi_{1}=\alpha=0^{\circ}$ (upper panel) and $60^{\circ}$ (lower panel), which correspond to the fields presented in Fig. 2. The mentioned asymmetry is clearly visible in the plateau region. The $\varepsilon_{n}=-1$ harmonics are dominant for $\varphi_{1}=0^{\circ}$, while for $\varphi_{1}=60^{\circ}$ the situation is opposite and the $\varepsilon_{n}=+1$ harmonics are dominant. Comparing the middle and bottom panels of Fig. 3 we can find more regions with such asymmetry. For example, for harmonic order near 30th and the angle $\varphi_{1} \approx 30^{\circ}$ there is a large dip in the harmonic power for $\varepsilon_{n}=-1$ (middle panel), while for $\varepsilon_{n}=+1$ (bottom panel) there is a strong maximum. By inspecting Fig. 2 one can relate this change in the asymmetry with the above-discussed overlap of the electron density and the electric field.

How we can use the results of the present work for future experiments? First, the absence of the $n=3 q$ harmonics from the HHG spectra of planar molecules having $C_{3}$ symmetry can be used as a measure of the orientation of the molecules in the plane. The measured parameter can be the ratio of the harmonic power $P_{3 q} / P_{3 q \pm 1}$ (it is zero for perfectly oriented molecules). Second, for molecules which are already oriented in a plane one can record the harmonic power of harmonics having different helicities as a function of the angle $\varphi_{1}$. The rotation angle of the bicircular field can easily be controlled in an experiment by changing the relative phase of the field components (see, for example, the experimental realization in Ref. 44). From these spectra one can extract information about the dominant molecular orbitals. In Figs. 1 and 3 the results for the HOMOs are presented. Similar calculations can be done for other molecules and different molecular orbitals and be used for comparison with experiments.

In conclusion, high harmonics emitted by polyatomic molecules that obey the $C_{r+s}$ symmetry, exposed to an $r \omega-s \omega$ bicircular field with the polarization plane perpendicular to the molecular quantization axis, can only have the order $n=q(r+s) \pm r$ and the corresponding ellipticity $\varepsilon_{n}= \pm 1$. We illustrated this by the examples of the planar molecules $\mathrm{BH}_{3}$ and $\mathrm{BF}_{3}$, which obey the $C_{3}$ symmetry. For the $\mathrm{BF}_{3}$ molecule, similarly to atoms having a $p$ ground state, there is a strong asymmetry in the emission of high harmonics of opposite helicities. This asymmetry depends on the molecular orientation and may be used for its determination. As in Refs. 36, 38, 39 for atoms, this asymmetry can be used to generate isolated elliptically polarized attosecond pulses by polyatomic molecules.

We thank Wilhelm Becker for useful comments. Support by the Federal Ministry of Education and Science, Bosnia and Herzegovina is gratefully acknowledged. 
TABLE I: The equilibrium geometries for $\mathrm{BH}_{3}$ and $\mathrm{BF}_{3}$ molecules calculated using the GAMESS 55 .

\begin{tabular}{|c|c|c|c|}
\hline atom & $x(\AA)$ & $y(\AA)$ & $z(\AA)$ \\
\hline B & 0.0000 & 0.0000 & 0.0000 \\
\hline H & -0.5937 & 1.0283 & 0.0000 \\
\hline H & -0.5937 & -1.0283 & 0.0000 \\
\hline H & 1.1874 & 0.0000 & 0.0000 \\
\hline
\end{tabular}

\begin{tabular}{|c|c|c|c|}
\hline atom & $x(\AA)$ & $y(\AA)$ & $z(\AA)$ \\
\hline $\mathrm{B}$ & 0.0000 & 0.0000 & 0.0000 \\
\hline $\mathrm{F}$ & -0.6473 & 1.1212 & 0.0000 \\
\hline $\mathrm{F}$ & -0.6473 & -1.1212 & 0.0000 \\
\hline $\mathrm{F}$ & 1.2947 & 0.0000 & 0.0000 \\
\hline
\end{tabular}

\section{Supplementary information}

\section{Molecular characteristics}

In this Supplementary Information, we show the equilibrium geometries, symmetries, and energies of the molecular orbitals for $\mathrm{BH}_{3}$ and $\mathrm{BF}_{3}$ molecules. The presented results are calculated using the Hartree-Fock method with cc-pVTZ basis set from the GAMESS quantum chemistry package 55 .

The $\mathrm{BH}_{3}$ and $\mathrm{BF}_{3}$ molecules correspond to the group $D_{3 h}$ which means that they have a $C_{3}$ axis, three $C_{2}$ axes and a $\sigma_{h}$ symmetry plane perpendicular to the $C_{3}$ axis. They also have three vertical planes of symmetry, each such plane passing through the $C_{3}$ axis and a $C_{2}$ axis. The results for the equilibrium geometries are shown in Table I] while the symmetries and energies of the molecular orbitals are presented in Table II.

\section{Definition of rotation}

Molecules are defined in a fixed Cartesian $x y z$ coordinate system. Passive rotation by the Euler angles of this system makes the $x$ axis in the direction $\hat{\mathbf{e}}_{1}$ ( $x$ axis of the bicircular field) and the $y$ axis in the direction $\hat{\mathbf{e}}_{2}$ ( $y$ axis of the bicircular field). According to [56], the product of three Euler rotations is $M(\alpha \beta \gamma)=M(\gamma) M(\beta) M(\alpha)$, where $\alpha$ denotes rotation about the original $z$ axis, $\beta$ about the new $y$ axis, and $\gamma$ about the final $z$ axis. In accordance with the above definition we have $M(\alpha \beta \gamma)(1,0,0)^{T}=\hat{\mathbf{e}}_{1}=$ $\left(\sin \theta_{1} \cos \varphi_{1}, \sin \theta_{1} \sin \varphi_{1}, \cos \theta_{1}\right)^{T}, M(\alpha \beta \gamma)(0,1,0)^{T}=$ $\hat{\mathbf{e}}_{2}=\left(\sin \theta_{2} \cos \varphi_{2}, \sin \theta_{2} \sin \varphi_{2}, \cos \theta_{2}\right)^{T}$ (the superscript $T$ indicates the transpose matrix), where the angles $\left(\theta_{1}, \varphi_{1}\right)$ and $\left(\theta_{2}, \varphi_{2}\right)$, respectively, define the vectors $\hat{\mathbf{e}}_{1}$ and $\hat{\mathbf{e}}_{2}$ in the spherical coordinates of the molecular sys-
TABLE II: Symmetries and energies of the $\mathrm{BH}_{3}$ and $\mathrm{BF}_{3}$ molecular orbitals. The cases of two degenerate orbitals are denoted by HOMOs, HOMO-1s etc.

\begin{tabular}{|c|c|c|}
\hline $\mathrm{BH}_{3}$ orbital & symmetry & energy $(\mathrm{eV})$ \\
\hline HOMO-1 & $A_{1}^{\prime}$ & -19.19 \\
\hline HOMOs & $E^{\prime}$ & -13.58 \\
\hline
\end{tabular}

\begin{tabular}{|c|c|c|}
\hline $\mathrm{BF}_{3}$ orbital & symmetry & energy $(\mathrm{eV})$ \\
\hline HOMO-6s & $E^{\prime}$ & -44.83 \\
\hline HOMO-5 & $A_{1}^{\prime}$ & -23.40 \\
\hline HOMO-4s & $E^{\prime}$ & -22.24 \\
\hline HOMO-3 & $A_{2}^{\prime \prime}$ & -20.88 \\
\hline HOMO-2s & $E^{\prime}$ & -19.08 \\
\hline HOMO-1s & $E^{\prime \prime}$ & -18.69 \\
\hline HOMO & $A_{2}^{\prime}$ & -17.99 \\
\hline
\end{tabular}

tem.

If the bicircular field polarization plane is the $x y$ plane then we have $\varphi_{1}=\varphi_{2}-90^{\circ}=\alpha$ and $\theta_{1}=\theta_{2}=90^{\circ}$, $\beta=\gamma=0^{\circ}$. For the field in the $x z$ plane we have $\alpha=$ $-\theta_{1}, \beta=\gamma=90^{\circ}$. In this case for $\theta_{1} \in\left[0^{\circ}, 90^{\circ}\right]$ we have $\varphi_{1}=\varphi_{2}=0^{\circ}, \theta_{2}=\theta_{1}+90^{\circ}$, while for $\theta_{1} \in\left[90^{\circ}, 180^{\circ}\right]$ it is $\varphi_{1}=0, \varphi_{2}=180^{\circ}, \theta_{2}=270^{\circ}-\theta_{1}$.

[1] F. Krausz and M. Ivanov, Attosecond physics, Rev. Mod. Phys. 81, 163 (2009).

[2] P. Agostini and L. F. DiMauro, Atomic and molecular ionization dynamics in strong laser fields: from optical to x-rays, Adv. At. Mol. Opt. Phys. 61, 117 (2012).

[3] W. Becker, X. Liu, P. J. Ho, and J. H. Eberly, Theories of photoelectron correlation in laser-driven multiple atomic ionization, Rev. Mod. Phys. 84, 1011 (2012).

[4] M. Kohler, T. Pfeifer, K. Hatsagortsyan, and C. Keitel, Frontiers of atomic high-harmonic generation, Adv. At. Mol. Opt. Phys. 61, 159 (2012).

[5] M. Lein, Molecular imaging using recolliding electrons, J. Phys. B 40, R135 (2007).

[6] C. D. Lin, A.-T. Le, Z. Chen, T. Morishita, and R. Lucchese, Strong-field rescattering physics - self-imaging of a molecule by its own electrons, J. Phys. B 43, 122001 (2010).

[7] N. L. Wagner, A. Wüest, I. P. Christov, T. Popmintchev, X. Zhou, M. M. Murnane, and H. C. Kapteyn, Monitoring molecular dynamics using coherent electrons from high harmonic generation, Proc. Natl. Acad. Sci. USA 103, 13279 (2006).

[8] R. Torres, N. Kajumba, J. G. Underwood, J. S. Robinson, S. Baker, J. W. G. Tisch, R. de Nalda, W. A. Bryan, R. Velotta, C. Altucci, I. C. E. Turcu, and J. P. Marangos, Probing Orbital Structure of Polyatomic Molecules by High-Order Harmonic Generation, Phys. Rev. Lett. 98, 203007 (2007). 
[9] W. Li, X. Zhou, R. Lock, S. Patchkovskii, A. Stolow, H. C. Kapteyn, and M. M. Murnane, Time-resolved dynamics in $\mathrm{N}_{2} \mathrm{O}_{4}$ probed using high harmonic generation, Science 322, 1207 (2008).

[10] M. C. H. Wong, J.-P. Brichta, and V. R. Bhardwaj, Signatures of symmetry and electronic structure in high-order harmonic generation in polyatomic molecules, Phys. Rev. A 81, 061402(R) (2010).

[11] M. C. H. Wong, J.-P. Brichta, M. Spanner, S. Patchkovskii, and V. R. Bhardwaj, High-harmonic spectroscopy of molecular isomers, Phys. Rev. A 84, 051403 (2011).

[12] C. Vozzi, M. Negro, F. Calegari, G. Sansone, M. Nisoli, S. De Silvestri, and S. Stagira, Generalized molecular orbital tomography, Nat. Phys. 7, 822 (2011).

[13] M. C. H. Wong, A. T. Le, A. F. Alharbi, A. E. Boguslavskiy, R. R. Lucchese, J.-P. Brichta, C. D. Lin, and V. R. Bhardwaj, High Harmonic Spectroscopy of the Cooper Minimum in Molecules, Phys. Rev. Lett. 110, 033006 (2013).

[14] A. T. Le, R. R. Lucchese, and C. D. Lin, Quantitative rescattering theory of high-order harmonic generation for polyatomic molecules, Phys. Rev. A 87, 063406 (2013).

[15] A. Ferré et al., Multi-channel electronic and vibrational dynamics in polyatomic resonant high-order harmonic generation, Nat. Commun. 6, 6592 (2015).

[16] P. M. Kraus, O. I. Tolstikhin, D. Baykusheva, A. Rupenyan, J. Schneider, C. Z. Bisgaard, T. Morishita, F. Jensen, L. B. Madsen, and H. J. Wörner, Observation of laser-induced electronic structure in oriented polyatomic molecules, Nat. Commun. 6, 8039 (2015).

[17] B. Manschwetus et al., Self-Probing Spectroscopy of the SF6 Molecule: A Study of the Spectral Amplitude and Phase of the High Harmonic Emission, J. Phys. Chem. A 119, 6111 (2015).

[18] P. Sándor, V. Tagliamonti, A. Zhao, T. Rozgonyi, M. Ruckenbauer, P. Marquetand, and T. Weinacht, Strong Field Molecular Ionization in the Impulsive Limit: Freezing Vibrations with Short Pulses, Phys. Rev. Lett. 116, 063002 (2016).

[19] S. Odžak, E. Hasović, and D. B. Milošević, Strong-fieldapproximation theory of high-order harmonic generation by polyatomic molecules, Phys. Rev. A 93, 043413 (2016).

[20] S. Odžak and D. B. Milošević, Bicircular-laser-fieldassisted electron-ion radiative recombination, Phys. Rev. A 92, 053416 (2015).

[21] H. Eichmann, A. Egbert, S. Nolte, C. Momma, B. Wellegehausen, W. Becker, S. Long, and J. K. McIver, Polarization-dependent high-order two-color mixing, Phys. Rev. A 51, R3414 (1995).

[22] S. Long, W. Becker, and J. K. McIver, Model calculations of polarization-dependent two-color high-harmonic generation, Phys. Rev. A 52, 2262 (1995).

[23] T. Zuo and A. D. Bandrauk, High-order harmonic generation in intense laser and magnetic fields, J. Nonlinear Opt. Phys. Mat. 04, 533 (1995).

[24] O. E. Alon, V. Averbukh, and N. Moiseyev, Selection Rules for the High Harmonic Generation Spectra, Phys. Rev. Lett. 80, 3743 (1998).

[25] W. Becker, B. N. Chichkov, and B. Wellegehausen, Schemes for the generation of circularly polarized highorder harmonics by two-color mixing, Phys. Rev. A 60, 1721 (1999).
[26] D. B. Milošević, W. Becker, and R. Kopold, Generation of circularly polarized high-order harmonics by two-color coplanar field mixing, Phys. Rev. A 61, 063403 (2000); D. B. Milošević and W. Sandner, Extreme-ultraviolet harmonic generation near $13 \mathrm{~nm}$ with a two-color elliptically polarized laser field, Opt. Lett. 25, 1532 (2000); D. B. Milošević, W. Becker, R. Kopold, and W. Sandner, High-harmonic generation by a bichromatic bicircular laser field, Laser Phys. 11, 165 (2001).

[27] D. B. Milošević and W. Becker, Attosecond pulse trains with unusual nonlinear polarization, Phys. Rev. A 62, 011403(R) (2000); Attosecond pulse generation by bicircular fields: from pulse trains to a single pulse, J. Mod. Opt. 52, 233 (2005).

[28] D. B. Milošević, W. Becker, and R. Kopold, Highharmonic generation by two-color circularly polarized field mixing, in Atoms, Molecules and Quantum Dots in Laser Fields: Fundamental Processes, Conference Proceedings Vol. 71, edited by N. Bloembergen, N. Rahman, and A. Rizzo (Società Italiana di Fisica, Bologna, 2001) pp. 239-252.

[29] F. Ceccherini, D. Bauer, and F. Cornolti, Dynamical symmetries and harmonic generation, J. Phys. B. 34, 5017 (2001).

[30] H. M. Nilsen, L. B. Madsen, and J. P. Hansen, On selection rules for atoms in laser fields and high harmonic generation, J. Phys. B. 35, L403 (2002).

[31] A. D. Bandrauk and H. Z. Lu, Controlling harmonic generation in molecules with intense laser and static magnetic fields: orientation effects, Phys. Rev. A 68, 043408 (2003).

[32] A. Fleischer, O. Kfir, T. Diskin, P. Sidorenko, and O. Cohen, Spin angular momentum and tunable polarization in high-harmonic generation, Nat. Photonics 8, 543 (2014).

[33] E. Pisanty and M. Ivanov, Taking control of polarization, Nat. Photonics 8, 501 (2014).

[34] E. Pisanty, S. Sukiasyan, and M. Ivanov, Spin conservation in high-order-harmonic generation using bicircular fields, Phys. Rev. A 90, 043829 (2014).

[35] O. Kfir et al., Generation of bright phase-matched circularly-polarized extreme ultraviolet high harmonics, Nat. Photonics 9, 99 (2015).

[36] D. B. Milošević, Generation of elliptically polarized attosecond pulse trains, Opt. Lett. 40, 2381 (2015).

[37] D. B. Milošević, High-order harmonic generation by a bichromatic elliptically polarized field: conservation of angular momentum, J. Phys. B 48, 171001 (2015).

[38] L. Medišauskas, J. Wragg, H. van der Hart, and M. Yu. Ivanov, Generating isolated elliptically polarized attosecond pulses using bichromatic counterrotating circularly polarized laser fields, Phys. Rev. Lett. 115, 153001 (2015).

[39] D. B. Milošević, Circularly polarized high harmonics generated by a bicircular field from inert atomic gases in the $p$ state: A tool for exploring chirality-sensitive processes, Phys. Rev. A 92, 043827 (2015).

[40] T. Fan et al., Bright circularly polarized soft X-ray high harmonics for X-ray magnetic circular dichroism, Proc. Natl. Acad. Sci. USA 112, 14206 (2015).

[41] C. Chen et al., Tomographic reconstruction of circularly polarized high-harmonic fields: 3D attosecond metrology, Sci. Adv. 2, e1501333 (2016).

[42] D. M. Reich and L. B. Madsen, Rotating-frame perspec- 
tive on high-order-harmonic generation of circularly polarized light, Phys. Rev. A 93, 043411 (2016).

[43] E. Hasović, D. B. Milošević, and W. Becker, A method of carrier-envelope phase control for few-cycle laser pulses, Laser Phys. Lett. 3, 200 (2006); A. Kramo, E. Hasović, D. B. Milošević, and W. Becker, Above-threshold detachment by a two-color bicircular laser field, Laser Phys. Lett. 4, 279 (2007); E. Hasović, A. Kramo, and D. B. Milošević, Energy- and angle-resolved photoelectron spectra of above-threshold ionization and detachment, Eur. Phys. J. Special Topics 160, 205 (2008).

[44] C. A. Mancuso et al., Strong-field ionization with twocolor circularly polarized laser fields, Phys. Rev. A 91, 031402(R) (2015).

[45] E. Hasović, W. Becker, and D. B. Milošević, Electron rescattering in a bicircular laser field, Opt. Express 24, 6413 (2016).

[46] C. A. Mancuso et al., Controlling electron-ion rescattering in two-color circularly polarized femtosecond laser fields, Phys. Rev. A 93, 053406 (2016).

[47] D. B. Milošević and W. Becker, Improved strong-field approximation and quantum-orbit theory: Application to ionization by a bicircular laser field, Phys. Rev. A 93, 063418 (2016).

[48] J. L. Chaloupka and D. D. Hickstein, Dynamics of Strong-Field Double Ionization in Two-Color Counterrotating Fields, Phys. Rev. Lett. 116, 143005 (2016).
[49] D. B. Milošević, Possibility of introducing spin into attoscience with spin-polarized electrons produced by a bichromatic circularly polarized laser field, Phys. Rev. A 93, 051402(R) (2016).

[50] D. Baykusheva, M. S. Ahsan, N. Lin, and H. J. Wörner, Bicircular High-Harmonic Spectroscopy Reveals Dynamical Symmetries of Atoms and Molecules, Phys. Rev. Lett. 116, 123001 (2016).

[51] F. Mauger, A. D. Bandrauk, and T. Uzer, Circularly polarized molecular high harmonic generation using a bicircular laser, J. Phys. B 49, 10LT01 (2016).

[52] H. Du, J. Zhang, S. Ben, H.-Y. Zhong, T.-T. Xu, J. Guo, and X.-S. Liu, High-order harmonic generation of $\mathrm{N}_{2}$ molecule in two-color circularly polarized laser fields, Chin. Phys. B 25, 043202 (2016).

[53] I. N. Levine, Quantum Chemistry, 5th ed. (Prentice Hall, New Jersey, 2000).

[54] D. S. Schonland, Molecular symmetry: an introduction to group theory and its uses in chemistry (Van Nostrand, London, 1965); Secs. 7.1-7.3.

[55] M. W. Schmidt et al., General Atomic and Molecular Electronic Structure System, J. Comput. Chem. 14, 1347 (1993).

[56] M. E. Rose, Elementary Theory of Angular Momentum (Dover, New York, 1995). 